\title{
A BOREL INVARIANTIZATION
}

\author{
BY ROBERT VAUGHT ${ }^{1}$
}

Communicated by J. Feldman, April 10, 1973

Let $G$ be a nonmeager topological group having a countable basis, and suppose $G$ acts on a topological space $X$. We show in $\S 1$ that for each Borel set $B \subseteq X$ there is an invariant Borel set $B^{*}$ lying between the usual invariantizations

$$
B^{-}=\{x: \forall g(g x \in B)\} \quad \text { and } \quad B^{+}=\{x: \exists g(g x \in B)\} .
$$

Moreover, the map $B \mapsto B^{*}$ has other regularities. Using $\S 1$, we establish in $\$ 2$ invariant versions for some classical theorems. For example, we show: Every invariant analytic set is the union of $\aleph_{1}$ invariant Borel sets. The transform * is applied to infinitary logic in $§ 3$. We give a new proof of the theorem: "invariant Borel $=L_{\omega_{1} \omega}$ ", and obtain several new theorems of the same form.

A detailed presentation will appear in Fundamenta Mathematica.

1. A transform. We assume the action $(g, x) \mapsto g x \in X$ is continuous in each variable separately (and, as usual, that $e x=x$ and $g(h x)=(g h) x$ ). Suppose $\mathscr{H}$ is a family of nonempty open sets such that any nonempty open set includes a member of $\mathscr{H}$. (The existence of a countable $\mathscr{H}$ is a sufficient countability assumption.) Below " $U$ ", " $V$ " always denote members of $\mathscr{H}$ and similarly $x \in X, g \in G, B \subseteq X, \alpha<\omega_{1}$, and $F: \omega \rightarrow \omega$. (A) is the smallest family containing all open sets and closed under complement, countable union, and the operation $(A)$. (For $(A)$ and other notions below, see [3].)

Definition 1.1. (a) $B^{x}=\left\{g: g^{-1} x \in B\right\}$.

(b) $B^{*}=\left\{x: B^{x}\right.$ is comeager in $\left.G\right\}$; and in general, $B_{U}^{*}=\left\{x: B^{x} \cap U\right.$ is comeager in $U\}$.

THEOREM 1.2. (a) $B_{U}^{*}$ is closed if $B$ is closed.

(b) $\left(\bigcap_{n} B_{n}\right)_{U}^{*}=\bigcap_{n}\left(B_{n}\right)_{U}^{*}$.

(c) $(\sim B)_{U}^{*}=\sim \bigcup^{n}\left\{B_{V}^{*}: V \subseteq U\right\}$.

(d) $x \in\left(\bigcup_{F} \bigcap_{n} B_{F \uparrow n}\right)_{U}^{*}$ if and only if

$$
\begin{aligned}
&\left(\forall U_{0} \subseteq U\right)\left(\exists V_{0} \subseteq U_{0}\right)\left(\exists k_{0}\right) \\
&\left(\forall U_{1} \subseteq V_{0}\right)\left(\exists V_{1} \subseteq U_{1}\right)\left(\exists k_{1}\right) \cdots \forall n\left[x \in\left(B_{k_{0} \cdots k_{n}}\right)_{V_{n}}^{*}\right] .
\end{aligned}
$$

The left side of (d) is the operation (A), while the right side denotes an

AMS (MOS) subject classifications (1970). Primary 02B25, 54H05, 54H15.

${ }^{1}$ Partially supported by NSF Grant GP-24352. 
ordinary infinite (closed) game-here a special case of $(A)$. (Compare the Banach-Mazur game [5].) In (c) and (d) it is assumed that $B$ (or the $\left.B_{k_{0} \cdots k_{n}}\right)$ is (A), or at least that each $B^{x}$ has the Baire property. Put $B^{\Delta}=\sim(\sim B)^{*}$.

THEOREM 1.3. (a) $B^{-} \subseteq B^{*} \subseteq B^{\Delta} \subseteq B^{+}$; and $B^{*}$ and $B^{\Delta}$ are invariant.

(b) $B^{*}$ is Borel or (A), if $B$ is.

(c) $B^{*}\left(\right.$ or $\left.B^{\Lambda}\right)$ is $\bigwedge_{\alpha}\left(\bigvee_{\alpha}\right)$-i.e., of Borel multiplicative (additive class) $\alpha$-provided $B$ is.

COROLlaRY 1.4. If $Y$ is an invariant subspace of $X$ and $B$ is $G_{\delta}$ (Borel, etc.) in $Y$, then $B=C \cap Y$ for some invariant $G_{\delta}($ etc.) set $C$.

Suppose now that $G^{\prime}$ is only a topological space (still Baire, with countable basis, but not a group). Let $D \subseteq G^{\prime} \times X$. Then we may consider $D^{*}=\left\{x:\left\{g^{\prime}:\left(g^{\prime}, x\right) \in D\right\}\right.$ is comeager $\}$; and similarly, $D_{U}^{*}, D^{-}=\{x$ : $\left.\forall g^{\prime}\left(\left(g^{\prime}, x\right) \in D\right)\right\}$, etc. The above still works, so that

TheOREM 1.5. If $D$ is Borel (A, etc.), so is $D^{*}$, and $D^{-} \subseteq D^{*} \subseteq D^{+} .^{2}$

Note also that 1.3(a), (b), 1.4 (and 2.1, 2.2 below) do not fully require that $X$ be a topological space. It suffices for $X$ to be a Borel space (Borel) acted upon by the same $G$, provided that some substitute for 1.2(a) is available.

Now we return to our basic assumptions.

2. Analytic sets. Suppose $E \subseteq X$ and $E=\bigcup_{F} \bigcap_{n} A_{F \uparrow n}$, where each $A_{k_{0} \cdots k_{n}}$ is, say, (A) (if they are all Borel, $E$ will be called analytic). Then there are canonical decompositions (in exactly the notation of $[3$, p. 32]):

$$
E=\bigcap_{\alpha} E_{\alpha}=\bigcup_{\alpha}\left(E_{\alpha}-T_{\alpha}\right) \text {. }
$$

THEOREM 2.1. $E^{*}=\bigcap_{\alpha} E_{\alpha}^{*}=\bigcup_{\alpha}\left(E_{\alpha}^{*} \cap\left(\sim T_{\alpha}\right)^{*}\right)$. Also, $E_{\alpha}^{*} \cap\left(\sim T_{\alpha}\right)^{*}$ is eventually comeager in $E^{*}$, if $X$ has a countable basis.

Taking $E=E^{*}$ we obtain

THEOREM 2.2. Every invariant analytic set is the union of $\aleph_{1}$ invariant Borel sets.

Assume $G$ is separable, complete metrizable. If $X$ is metrizable, then 2.2 with " $\aleph_{1}$ " omitted coincides with the known theorem: Every orbit ( $\{g x: g \in G\})$ is Borel. (Ryll-Nardzewski [7], extending a theorem of Scott; A related result is in Dixmier [1].)

${ }^{2}$ Theorem 1.5 for Borel (or lower) sets was proved by P. S. Novikov for the case $X=$ $G^{\prime}=$ the unit interval, by a similar argument. See W. J. Arsenin, A. A. Ljapunow, and E. A. Stschegolkow, Arbeiten zur Deskriptives Mengenlehre, VEB Deutsches Verlag des Wiss., Berlin, 1955, or Uspehi Mat. Neuk SSSR 39 (1950), 11-119. (Russian). 
Now assume $X$ is separable, complete metrizable. Then 2.2 extends at once to invariant PCA $\left(\Sigma_{2}\right)$ sets. Moreover,

THEOREM 2.3. The reduction principle (cf. [3]) applies to "invariant coanalytic" and to "invariant PCA."

The invariant separation principle

disjoint invariant analytic sets can be separated by an invariant Borel set

can be proved by a direct ( $\omega$-union) argument, as Ryll-Nardzewski noted years ago. As is known, a weak invariant covering theorem (see [3]) can be similarly obtained. 2.1 implies a more explicit covering theorem.

2.2 and 2.3 were already established in the logic spaces (cf. §3) in [8], using work of Moschovakis.

3. Infinitary logic. Let $G=\omega$ !, the group of all permutations of $\omega$ (with the product topology). Let $X$ be a space such as $2^{\omega \times \omega \times \omega}$, whose members can be considered as ternary relations $R$ or as structures $(\omega, R) . g R$ is the usual isomorph of $R$ under $g$.

The language $L_{\omega_{1} \omega}$ has atomic formulas $R u v w$ and $u=v$. Arbitrary formulas are obtained by (repeatedly) applying $\sim, \exists u$, and countably infinite disjunctions $V_{n} \phi_{n}$, where all the $\phi_{n}$ have the same finite list of free variables. Each sentence $\sigma$ can be identified with the class $B=$ Mod $\sigma \subseteq X$ of all models $R$ of $\sigma$. For a formula $\phi(u, v)$, for example, $\operatorname{Mod} \phi \subseteq X \times \omega \times \omega$.

There is a natural classification $\Lambda_{\alpha}^{\prime}\left(V_{\alpha}^{\prime}\right)$ of formulas in which, e.g., $\phi$ is $\bigwedge_{\alpha+1}^{\prime}$ if $\phi=\bigwedge_{n} \forall u_{1} \cdots u_{k_{n}} \psi_{n}$, where each $\psi_{n}$ is $\bigvee_{\alpha}^{\prime}$. Also, call $B \subseteq X$ meager' if $B \subseteq \operatorname{Mod}\left[\bigvee_{n} \exists u_{1} \cdots u_{k_{n}} \phi_{n}\right]$ where each Mod $\phi_{n}$ is closed, nowhere dense. The notion meager' occurs implicitly in the $\omega$-completeness or omitting types theorem (see, e.g., [2]).

If $s$ is a finite sequence of distinct natural numbers, put $[s]=\{g: s \subseteq g\}$. Let $\mathscr{H}$ be the basis consisting of all [s]. Now 1.2(b), for example, reads: $(\sim \sigma)^{*} \Leftrightarrow(\exists s) \sigma_{[s]}^{*}$. Thus the whole right side of 1.2 takes place in quantifier logic (since, as is well known and simple, invariant closed $=\Lambda_{0}^{\prime}$ ). In this way the following results are obtained:

THEOREM 3.1 (LOPEZ-EsCOBAR [4]). Invariant Borel $=($ expressible in $)$ $L_{\omega_{1} \omega}$.

3.1 was inferred by Scott from Lopez-Escobar's Interpolation Theorem (IT). However, as Ryll-Nardzewski remarked at the time, IT can also be inferred from 3.1, by (1) above. Thus 3.1 gives the first proof of IT from the classical first separation principle. 
By the new proof (or 1.4), 3.1 can now be seen to hold relativized to any invariant $Y \subseteq X$ (and the same applies to 3.2-3.5 below).

THEOREM 3.2. Invariant $\Lambda_{\alpha}\left(\mathrm{V}_{\alpha}\right)=\Lambda_{\alpha}^{\prime}\left(\mathrm{V}_{\alpha}^{\prime}\right)$.

THEOREM 3.3. Invariant meager $=$ meager'

The language $L_{G}$ has the formation rules of $L_{\omega_{1} \omega}$ plus the use of the infinite closed game quantifier

$$
\forall u_{0} \exists v_{0} \exists k_{0} \forall u_{1} \exists v_{1} \exists k_{1} \cdots \bigwedge_{n} \phi^{k_{0} \cdots k_{n}}\left(u_{0}, v_{0}, \ldots, u_{n}, v_{n}, w_{0}, \ldots, w_{m-1}\right) .
$$

$L_{G}$ has been studied by Moschovakis and by Barwise.

Using $1.2(\mathrm{~d})$ it is easy to show:

\section{THEOREM 3.4. Invariant $(A)=L_{G}$.}

Finally, let $\kappa$ be an infinite regular cardinal and form $\kappa$ ! and, say, $2^{\kappa \times \kappa \times \kappa}$, each with the $\kappa$-topology (induced by intersections of fewer than $\kappa$ product open sets). $\kappa$-Borel sets are formed using complements and $\kappa$-unions. $L_{\kappa+\kappa}$ is just like $L_{\omega_{1} \omega}$ but allows $\kappa$-disjunctions and infinite quantifications $\left(\exists u_{0} \cdots u_{\xi} \cdots\right)_{\xi<\gamma}$, where $\gamma<\kappa$. Since $\kappa$ ! is a " $\kappa$-Baire" topological group (cf., e.g., [6]) we can repeat $\$ 1$ in the $\kappa$-topology, obtaining

THEOREM 3.5. Invariant $\kappa$-Borel $=L_{\kappa^{+}}$, provided $2^{\kappa}=\kappa$.

\section{BIBLIOGRAPHY}

1. J. Dixmier, Duel et quasi-duel d'une algèbre de Banach involutive, Trans. Amer. Math. Soc. 104 (1962), 278-283. MR 25 \#3384.

2. A. Grzegorczyk, A. Mostowski, and C. Ryll-Nardzewski, Definability of sets in models of axiomatic theories, Bull. Acad. Polon. Sci. Sér. Sci. Math. Astronom. Phys. 9 (1961), 163-167. MR 29 \#1138.

3. K. Kuratowski, Topologie. Vol. I, 2nd ed., Monografie Mat., Tom 20, WarszawaWrocław, 1948; English transl., New ed., rev. and aug., Academic Press, New York, 1966. MR 10, 389; 36 \# 840 .

4. E. G. K. Lopez-Escobar, An interpolation theorem for denumerably long formulas, Fund. Math. 57 (1965), 253-272. MR 32 \#5500.

5. J. Mycielski, S. Swierczkowski and A. Zieba, On infinite positional games, Bull. Acad. Polon. Sci. Cl. III. 4 (1956), 485-488. MR 19, 232.

6. G. E. Reyes, Local definability theory, Ann. Math. Logic 1 (1970), 95-137. MR 43 \# 31.

7. C. Ryll-Nardzewski, On Borel measurability of orbits, Fund. Math. 56 (1964), 129-130. MR 30 \# 3028.

8. R. Vaught, Descriptive set theory in $L_{\omega_{1} \omega}$, Proc. Sympos. (Cambridge University, 1971) (to appear).

Department of Mathematics, University of California, Berkeley, California 94720 